\title{
Crystal Structure of Acenaphthene-1'-one-spiro[2'.3]-[4-ethoxycarbonyl]- $N$ - methyl-pyrrolidine-spiro[2.2"]-acenaphthene-1"-one
}

\author{
G. Usha, $*$ S. Selvanayagam, $*$ D. Velmurugan, $* \dagger$ K. Ravikumar, $* *$ J. Jayashankaran, $* * *$ \\ R. RAGHUNATHAN $* * *$ \\ *Department of Crystallography and Biophysics, University of Madras, Guindy Campus, \\ Chennai-600 025, India \\ **Laboratory of X-ray crystallography, Indian Institute of Chemical Technology, Hyderabad-500 007, India \\ ***Department of Organic Chemistry, University of Madras, Guindy Campus, Chennai-600 025, India
}

\begin{abstract}
In the title compound, $\mathrm{C}_{30} \mathrm{H}_{23} \mathrm{NO}_{4}$, the molecule includes two spiro junctions connecting acenaphthene rings and the pyrrolidine moiety. The acenaphthene ring atoms $(\mathrm{C} 2, \mathrm{C} 7-\mathrm{C} 17)$ and $(\mathrm{C} 3, \mathrm{C} 18-\mathrm{C} 28)$ make dihedral angles of 87.0(1) and $81.3(1)^{\circ}$, respectively, with the pyrrolidine ring system. The pyrrolidine ring exhibits an envelope conformation. In the crystal, packing is stabilized by $\mathrm{C}-\mathrm{H} \cdots \pi$ and $\pi-\pi$ interactions.
\end{abstract}

(Received May 11, 2005; Accepted October 25, 2005; Published on web February 13, 2006)

Spiro pyrrolidine derivatives are often used in the synthesis of biologically active compounds. The spiro ring system is also very interesting from a biogenetic point of view. Synthetic pyrrolidine derivatives have activity against the aldose reductase enzyme, which controls influenza. ${ }^{1}$ Acenaphthene of a known concentration added to soil samples helps one to investigate the anaerobic degradation potential of polycyclic aromatic hydrocarbons. ${ }^{2}$ Polycyclic azaarenes (PAA) are relatively rigid planar hosts for metal ions and organic molecules. $^{3}$ In view of its varied importance, crystal and molecular structure determinations of the title compound were carried out by X-ray diffraction (Fig. 1).

The crystal data were collected on a CCD Area Detector diffractomter (Table 1) with graphite-monochromated Mo $K_{\alpha}$ radiation. The structure was solved by direct methods and refined by a full-matrix least-squares procedure. The nonhydrogen atoms were refined anisotropically. The $\mathrm{H}$ atoms were positioned geometrically and were treated as riding on their parent $\mathrm{C}$ atoms and refined isotropically. The final atomic coordinates and equivalent isotropic thermal parameters are given in Table 2.

The selected bond lengths and bond angles are given in Table 3. The $\mathrm{C}-\mathrm{C}$ distances and $\mathrm{C}-\mathrm{C}-\mathrm{C}$ angles of the pyrrolidine

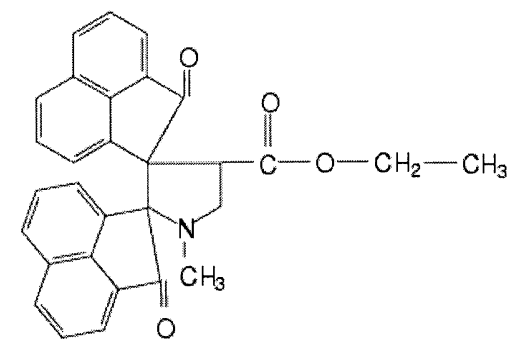

Fig. 1 Chemical diagram.

$\doteqdot$ To whom correspondence should be addressed.

E-mail: d_velu@yahoo.com moiety shows variations, which can be considered to be the characteristic feature of compounds containing spiro junctions. The endocyclic torsion angles show the five-membered ring in an envelope conformation with the $\mathrm{N} 1$ atom out of the plane of the four others, which are planar within $0.002 \AA$. The displacement of the N1 atom from the plane is $-0.611(2) \AA$. The sum of the angles around the N1 atom is $338.1(1)^{\circ}$, showing $\mathrm{sp}^{3}$ hybridization.

The bond lengths and angles of the acenaphthene rings of atoms $\mathrm{C} 2, \mathrm{C} 7-\mathrm{C} 17$ and $\mathrm{C} 3, \mathrm{C} 18-\mathrm{C} 28$ are in the same range, and closely resemble the values of the related structures reported so far. ${ }^{4}$ The acenaphthene rings are almost planar with the associated ketone atoms $\mathrm{O} 1$ and $\mathrm{O} 2$ displaced by $-0.207(2)$ and $-0.301(1) \AA$, respectively.

Table 1 Crystal and experimental data

\begin{tabular}{lll}
\hline CCDC No. & 270968 & \\
Empirical formula & $\mathrm{C}_{30} \mathrm{H}_{23} \mathrm{NO}_{4}$ & \\
Formula weight & 461.49 & \\
Wavelength & $0.71073 \AA$ & \\
Crystal system & triclinic & \\
Space group & $P \overline{1}$ & \\
Unit cell dimensions & $a=9.3442(8) \AA \quad \alpha=79.538(2)^{\circ}$ \\
& $b=10.3875(9) \AA \quad \beta=1.8570(10)^{\circ}$ \\
& $c=12.1667(10) \AA \quad \gamma=87.1640(10)^{\circ}$ \\
Volume & $1149.25(17) \AA^{3}$ & \\
$Z$ & 2 & \\
Density (calculated) & $1.334 \mathrm{Mg} / \mathrm{m}^{3}$ & \\
$\theta_{\text {max }}$ & $28^{\circ}$ & \\
No. of reflections used & 4574 & \\
$R$ & 0.0570 & \\
$(\Delta / \sigma)_{\max }$ & $<0.001$ & \\
$(\Delta \rho)_{\max }$ & $0.278 \mathrm{e} \AA^{-3}$ & \\
$(\Delta \rho)_{\min }$ & $-0.203 \mathrm{e} \AA^{-3}$ & \\
Measurement & CCD Area Detector diffractometer \\
Program system & SAINT & \\
Structure determination & SHELXS97 \\
Refinement method & Full-matrix least-squares \\
\hline
\end{tabular}


Table 2 Atomic coordinates $\left(\times 10^{4}\right)$ and equivalent isotropic displacement parameters $\left(\AA^{2} \times 10^{3}\right)$ for the non-hydrogen atoms

\begin{tabular}{|c|c|c|c|c|}
\hline Atom & $x$ & $y$ & $z$ & $* U_{(\mathrm{eq})}$ \\
\hline $\mathrm{O} 1$ & $853(2)$ & $9131(1)$ & $6488(2)$ & $66(1)$ \\
\hline $\mathrm{O} 2$ & $-511(2)$ & $6108(2)$ & 9874(1) & $56(1)$ \\
\hline $\mathrm{O} 3$ & $-1704(2)$ & $11010(2)$ & $8014(2)$ & $108(1)$ \\
\hline O4 & $-3738(2)$ & $9943(2)$ & $8597(2)$ & $84(1)$ \\
\hline N1 & $557(2)$ & $7525(1)$ & $8833(1)$ & $44(1)$ \\
\hline $\mathrm{C} 2$ & $-62(2)$ & $7207(2)$ & $7882(2)$ & $38(1)$ \\
\hline $\mathrm{C} 3$ & $-1697(2)$ & $7657(2)$ & $8140(2)$ & $38(1)$ \\
\hline $\mathrm{C} 4$ & $-1607(2)$ & $8738(2)$ & $8874(2)$ & $47(1)$ \\
\hline C5 & $-17(2)$ & $8819(2)$ & $8985(2)$ & $53(1)$ \\
\hline C6 & $2113(2)$ & $7355(2)$ & $8797(2)$ & $62(1)$ \\
\hline C7 & $642(2)$ & $7965(2)$ & $6704(2)$ & $44(1)$ \\
\hline $\mathrm{C} 8$ & $1074(2)$ & $7005(2)$ & $5954(2)$ & $42(1)$ \\
\hline C9 & $1660(2)$ & $7122(2)$ & $4849(2)$ & $55(1)$ \\
\hline $\mathrm{C} 10$ & $2011(2)$ & $5979(3)$ & $4402(2)$ & $64(1)$ \\
\hline $\mathrm{C} 11$ & $1800(2)$ & $4765(3)$ & $5047(2)$ & $59(1)$ \\
\hline $\mathrm{C} 12$ & $1213(2)$ & $4604(2)$ & $6193(2)$ & $46(1)$ \\
\hline $\mathrm{C} 13$ & $828(2)$ & $5759(2)$ & $6616(2)$ & $38(1)$ \\
\hline C14 & $206(2)$ & $5787(2)$ & $7730(2)$ & $36(1)$ \\
\hline $\mathrm{C} 15$ & $7(2)$ & $4627(2)$ & $8458(2)$ & $44(1)$ \\
\hline $\mathrm{C} 16$ & $384(2)$ & $3450(2)$ & $8044(2)$ & $54(1)$ \\
\hline $\mathrm{C} 17$ & $953(2)$ & $3420(2)$ & $6961(2)$ & $54(1)$ \\
\hline $\mathrm{C} 18$ & $-2581(2)$ & $6493(2)$ & $8887(2)$ & $39(1)$ \\
\hline C19 & $-3577(2)$ & $6065(2)$ & $8196(2)$ & $40(1)$ \\
\hline $\mathrm{C} 20$ & $-4526(2)$ & $5052(2)$ & $8405(2)$ & $49(1)$ \\
\hline $\mathrm{C} 21$ & $-5412(2)$ & $4969(2)$ & $7585(2)$ & $59(1)$ \\
\hline $\mathrm{C} 22$ & $-5377(2)$ & $5874(2)$ & $6615(2)$ & $59(1)$ \\
\hline $\mathrm{C} 23$ & $-4435(2)$ & $6946(2)$ & $6387(2)$ & $49(1)$ \\
\hline $\mathrm{C} 24$ & $-3526(2)$ & $6984(2)$ & $7204(2)$ & $40(1)$ \\
\hline $\mathrm{C} 25$ & $-2482(2)$ & $7957(2)$ & $7106(2)$ & $40(1)$ \\
\hline $\mathrm{C} 26$ & $-2366(2)$ & $8904(2)$ & $6166(2)$ & $53(1)$ \\
\hline $\mathrm{C} 27$ & $-3285(3)$ & $8895(2)$ & $5344(2)$ & $63(1)$ \\
\hline $\mathrm{C} 28$ & $-4293(2)$ & $7961(2)$ & $5444(2)$ & $62(1)$ \\
\hline $\mathrm{C} 29$ & $-2311(2)$ & $10030(2)$ & $8445(2)$ & $61(1)$ \\
\hline C30 & $-4576(4)$ & $11113(3)$ & $8207(4)$ & $123(2)$ \\
\hline $\mathrm{C} 31$ & $-5947(5)$ & $10792(4)$ & $8041(4)$ & $139(2)$ \\
\hline
\end{tabular}

$* U_{(\mathrm{eq})}=(1 / 3) \sum_{i} \Sigma_{j} U_{i j} a_{i} * a_{j}^{*}\left(\boldsymbol{a}_{i} \cdot \boldsymbol{a}_{j}\right)$.

Table 3 Selected bond lengths $[\AA]$ and angles $\left[^{\circ}\right]$

\begin{tabular}{|c|c|c|c|c|}
\hline $\mathrm{O} 1$ & $853(2)$ & 9131(1) & $6488(2)$ & $66(1)$ \\
\hline $\mathrm{O} 2$ & $-511(2)$ & $6108(2)$ & 9874(1) & $56(1)$ \\
\hline $\mathrm{O} 3$ & $-1704(2)$ & $11010(2)$ & $8014(2)$ & $108(1)$ \\
\hline $\mathrm{O} 4$ & $-3738(2)$ & $9943(2)$ & $8597(2)$ & $84(1)$ \\
\hline $\mathrm{N} 1$ & $557(2)$ & $7525(1)$ & $8833(1)$ & $44(1)$ \\
\hline $\mathrm{C} 2$ & $-62(2)$ & $7207(2)$ & $7882(2)$ & $38(1)$ \\
\hline C3 & $-1697(2)$ & $7657(2)$ & $8140(2)$ & $38(1)$ \\
\hline $\mathrm{C} 4$ & $-1607(2)$ & $8738(2)$ & $8874(2)$ & $47(1)$ \\
\hline C5 & $-17(2)$ & $8819(2)$ & $8985(2)$ & $53(1)$ \\
\hline C6 & $2113(2)$ & $7355(2)$ & $8797(2)$ & $62(1)$ \\
\hline C7 & $642(2)$ & $7965(2)$ & $6704(2)$ & $44(1)$ \\
\hline $\mathrm{C} 8$ & $1074(2)$ & $7005(2)$ & $5954(2)$ & $42(1)$ \\
\hline C9 & $1660(2)$ & $7122(2)$ & $4849(2)$ & $55(1)$ \\
\hline $\mathrm{C} 10$ & 2011(2) & $5979(3)$ & $4402(2)$ & $64(1)$ \\
\hline $\mathrm{C} 11$ & $1800(2)$ & $4765(3)$ & $5047(2)$ & $59(1)$ \\
\hline $\mathrm{C} 12$ & $1213(2)$ & $4604(2)$ & $6193(2)$ & $46(1)$ \\
\hline $\mathrm{C} 13$ & $828(2)$ & $5759(2)$ & $6616(2)$ & $38(1)$ \\
\hline $\mathrm{C} 14$ & $206(2)$ & $5787(2)$ & $7730(2)$ & $36(1)$ \\
\hline $\mathrm{C} 15$ & $7(2)$ & $4627(2)$ & $8458(2)$ & $44(1)$ \\
\hline $\mathrm{C} 16$ & $384(2)$ & $3450(2)$ & $8044(2)$ & $54(1)$ \\
\hline $\mathrm{C} 17$ & $953(2)$ & $3420(2)$ & $6961(2)$ & $54(1)$ \\
\hline $\mathrm{C} 18$ & $-2581(2)$ & $6493(2)$ & $8887(2)$ & $39(1)$ \\
\hline C19 & $-3577(2)$ & $6065(2)$ & $8196(2)$ & $40(1)$ \\
\hline $\mathrm{C} 20$ & $-4526(2)$ & $5052(2)$ & $8405(2)$ & $49(1)$ \\
\hline $\mathrm{C} 21$ & $-5412(2)$ & $4969(2)$ & $7585(2)$ & $59(1)$ \\
\hline $\mathrm{C} 22$ & $-5377(2)$ & $5874(2)$ & $6615(2)$ & $59(1)$ \\
\hline $\mathrm{C} 23$ & $-4435(2)$ & $6946(2)$ & $6387(2)$ & $49(1)$ \\
\hline $\mathrm{C} 24$ & $-3526(2)$ & $6984(2)$ & $7204(2)$ & $40(1)$ \\
\hline $\mathrm{C} 25$ & $-2482(2)$ & $7957(2)$ & $7106(2)$ & $40(1)$ \\
\hline $\mathrm{C} 26$ & $-2366(2)$ & $8904(2)$ & $6166(2)$ & $53(1)$ \\
\hline $\mathrm{C} 27$ & $-3285(3)$ & $8895(2)$ & $5344(2)$ & $63(1)$ \\
\hline $\mathrm{C} 28$ & $-4293(2)$ & $7961(2)$ & $5444(2)$ & $62(1)$ \\
\hline $\mathrm{C} 29$ & $-2311(2)$ & $10030(2)$ & $8445(2)$ & 61(1) \\
\hline C30 & $-4576(4)$ & $11113(3)$ & $8207(4)$ & $123(2)$ \\
\hline C31 & $-5947(5)$ & $10792(4)$ & $8041(4)$ & $139(2)$ \\
\hline
\end{tabular}

Table 4 Non-bonded interactions [ $\left[\AA\right.$ and ${ }^{\circ}$ ]

\begin{tabular}{lcccc}
\hline D-H...A & D-H & H...A & D...A & $<($ DHA $)$ \\
\hline C4-H4...O2 & 0.98 & 2.40 & $2.896(2)$ & 111 \\
C5-H5A...O1 & 0.97 & 2.41 & $2.995(3)$ & 118 \\
C5-H5A...O3 & 0.97 & 2.54 & $2.874(3)$ & 100 \\
C26-H26...O1 & 0.93 & 2.50 & $3.113(3)$ & 124 \\
\hline
\end{tabular}

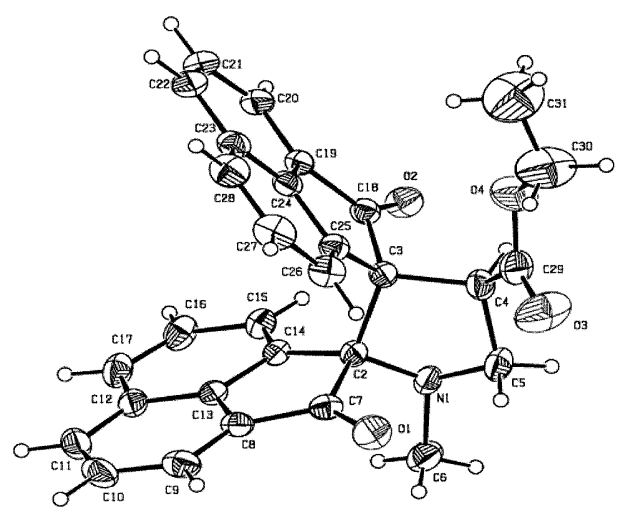

Fig. 2 Molecular structure of the title compound, showing 30\% probability displacement ellipsoids.

An ORTEP diagram of the molecule, togther with the atomic labelling scheme is shown in Fig. 2. Ethoxycarbonyl group atoms show large anistropic thermal motion. The molecule is stabilized by a number of $\mathrm{C}-\mathrm{H}$... $\mathrm{O}$ type intramolecular interactions. The $\mathrm{C}-\mathrm{H} \cdots \pi$ interaction between $\mathrm{C} 11$ and the ring (A) of atoms $(\mathrm{C} 23-\mathrm{C} 28)$ at $-x, 1-y, 1-z \quad[\mathrm{C} 11 \cdots \mathrm{CgA}=$ 3.642(3) $\AA$ : $\angle \mathrm{C} 11-\mathrm{H} 11 \cdots \mathrm{CgA}=167.2^{\circ}$; $\mathrm{CgA}$ is the centroid of ring $\mathrm{A}]$ and the $\pi-\pi$ interaction between ring (B) of atoms (C8-C13) at $x, y, z$ and $-x, 1-y, 1-z$ with centroid-centroid distance of $3.770(1) \AA$ are observed in the crystal. These interactions stabilize the crystal packing in addition to van der Waals interactions.

\section{Acknowledgements}

GU thanks the University Grants Commission (UGC) for an award of the Faculty Improvement Program (FIP). SS thanks the Council of Scientific and Industrial Research (CSIR) for providing a Senior Research Fellowship. DV acknowledges UGC and Department of Bio-Technology (DBT) for providing computing facilities under major Research projects and also gives thanks for the financial support to the Department under UGC-SAP and DST-FIST programs.

\section{References}

1. I. Stylianakis, A. Kolocouris, N. Kolocouris, G. Fytas, G. B. Foscolos, E. Padalko, J. Neyts, and E. Declercq, Bioorg. Med. Chem. Lett., 2003, 13, 1699.

2. B. V. Chang, L. C. Shiung, and S. Y. Yuan, Chemosphere, 2002, 48, 717 .

3. T. W. Bell and A. Firestone, J. Am. Chem. Soc., 1986, 108, 8190.

4. T. Repo, M. Klinga, M. Leskela, M. Polamo, and B. Rieger Acta Cryst., 1996, C52, 2910. 